\title{
Impact of micromechanical parameters on wire sawing: a 3D discrete element analysis
}

\author{
B. Nassauer ${ }^{1}$ - M. Kuna ${ }^{1}$
}

Received: 23 December 2014 / Revised: 26 March 2015 / Accepted: 31 March 2015 / Published online: 9 April 2015 (C) OWZ 2015

\begin{abstract}
Wire sawing is one of the most prominent technologies for producing solar wafers. In the following paper a numerical model for the simulation of the wire sawing process at microlevel using the discrete element method is presented. The abrasive particles are represented by convex polyhedra. Based on the contact forces on the ingot, local material removal rates are calculated. These local material removal rates are then used to update the shape of the ingot in a stepwise simulation. The model allows for detailed analysis of the influence of different parameters on the micromechanical processes during wire sawing. In particular the influence of wire speed, lapping pressure, particle concentration, particle size, particle shape and debris on the sawing process is investigated. In a post-processing macroscopic process characteristics like kerf width, depth of surface cracks or sawing rate are calculated. Thus, the processes at the microlevel are related to the macroscopic process characteristics, which are of interest when applying wire sawing in practice.
\end{abstract}

Keywords Wire sawing - DEM simulation - Polyhedral particles $\cdot$ Material removal

\section{Introduction}

Wire sawing is the usual process for sawing silicon ingots into wafers, which are used to produce solar cells or micro chips. Due to the high influence on the manufacturing costs

M. Kuna

meinhard.kuna@imfd.tu-freiberg.de

B. Nassauer

Benjamin.Nassauer@imfd.tu-freiberg.de

1 Institute of Mechanics and Fluid Dynamics, TU Bergakademie Freiberg, 09596 Freiberg, Germany of solar cells, the wire sawing process has been in the focus of research for several years. Numerous experimental and numerical investigations deal with the macroscopic aspects of wire sawing. Nevertheless detailed understanding of the processes at the micro level is still lacking. Ishikawa et al. [1], Kaminski et al. [2] and Liedke [3] performed experiments of sawing into glass in order to directly observe the sawing channel. Liedke [4] performed 2D DEM simulations, where the abrasive particles where represented by polygons. The material removal was modeled for every contact. Nevertheless the limitation to 2 dimensions is a great disadvantage of the model. 3D DEM simulations have been performed by Wagner and Möller [5] and Bierwisch et al. [6]. In these models the abrasive particles are represented by spheres or clusters of spheres, thus the sharp edges of the real abrasive particles are not represented. First 3D DEM simulations with polyhedral particles can be found in [7]. These polyhedral particles provide a very good representation of the shape of real abrasive particles, including the sharp edges. Nevertheless in all 3D DEM simulations no material removal was considered. This is a great disadvantage as the result depends on the choice of the ingot geometry.

In the present paper the wire sawing process is simulated at microlevel by 3D DEM simulations with polyhedral particles. The contact forces between ingot and abrasive particles are used to evaluate the material removal. In a stepwise simulation the ingot geometry is updated considering the calculated material removal. As the micromechanical processes are modeled in detail, the influence of different parameters on the behaviour at micro level can be investigated. In extensive simulations the influence of wire speed, lapping pressure, particle concentration, particle size, particle shape and debris is investigated. When applying wire sawing in practice, several macroscopic process parameters are of interest. The kerf width defines the amount of silicon which is lost during the 
process and has to be recovered in costly recycling processes. Thus, a small kerf width is desirable. After sawing, surface cracks remain in the wafer as sawing damage. The smaller the depth of these cracks the higher the stability of the wafer and the lower the work needed in further production steps. Furthermore, the sawing rates are of special interest. Due to the low sawing rates one cut takes several hours. Therefore, improvements in the sawing rate can lead to high savings in process time and thereby significantly reduce the production costs. These macroscopic process parameters are calculated from the simulation results in a post-processing routine. Thus, the microscopic effects are related to the macroscopic process. On the one hand the microscopic simulations can be verified by macroscopic experiments. On the other hand, the simulations on the micro level can help to explain macroscopic observations. Furthermore, the microscopic simulations can disclose possibilities to improve the macroscopic process. It is necessary to perform simulations since direct observation of the micromechanical mechanisms is rather difficult.

\section{Numerical model}

The wire sawing process is simulated by means of 3D discrete element method employing polyhedral particles. For details on DEM with this specific particle shape the reader is referred to [8] and [9]. The interaction between particles and fluid flow of the slurry is reported in [7] and [10]. In the following, only the most important facts are summarized: The influence of the fluid having a viscosity $\eta$ on the particle motion is estimated by Stokes law. For this, a particle is approximated by a sphere of radius $R$ surrounding the particle completely. Then, the force $F_{i}^{\mathrm{S}}$ acting on the particle is given as

$F_{i}^{\mathrm{S}}=6 \pi \eta R\left(v_{i}^{\mathrm{f}}-v_{i}\right)$,

where $v_{i}^{\mathrm{f}}$ is the fluid velocity at the particle position and $v_{i}$ denotes the particle velocity. A laminar plane Couette flow is assumed in the sawing channel between wire and ingot, which is orientated parallel to the wire. The fluid velocity takes the velocity $v_{i}^{\mathrm{w}}$ of the wire at its surface and decreases to zero towards the ingot surface. Thus, the fluid velocity is linearly interpolated across the channel width $h$ in radial direction to the wire. The corresponding shear rate is calculated by $\dot{\gamma}=\left|v_{i}^{\mathrm{W}}\right| / h$. In addition, a torque $M_{i}^{\mathrm{S}}$ is exerted on the particle, which is obtained by

$M_{i}^{\mathrm{S}}=8 \pi \eta R^{3}\left(\dot{\gamma}_{i}-\omega_{i}\right)$,

where $\dot{\gamma}_{i}$ is the shear rate vector of the fluid at the position of the particle, and $\omega_{i}$ is the angular velocity of the particle.

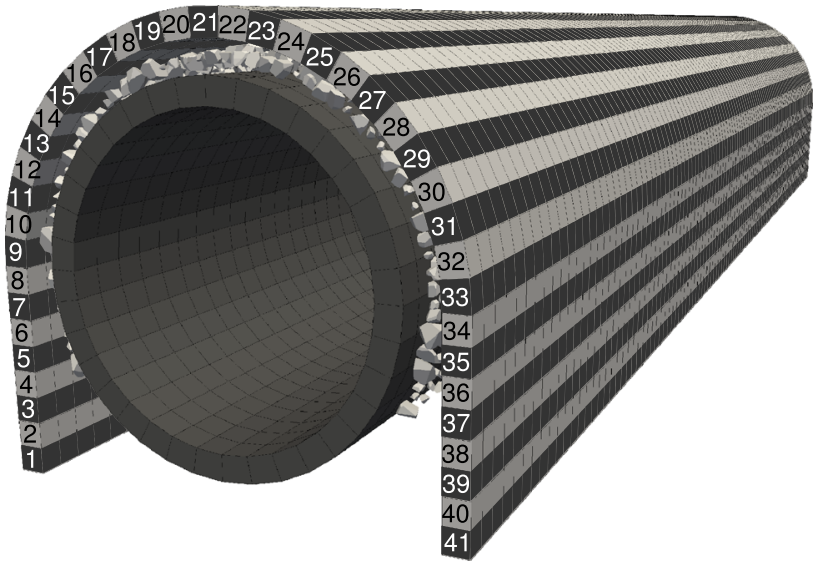

Fig. 1 Partitions of the ingot in which the material removal is evaluated

The feedback from the particle motion to the fluid flow is not considered.

The wire is simulated by an assembly of brick-like particles glued together as shown in Fig. 1. The movement of the wire in its axial direction is prescribed by the wire velocity, which is held constant throughout the simulation. In vertical direction, the wire is pressed against the ingot with a certain pressure, called lapping pressure $p$. In the numerical model the lapping pressure is directly applied by a defined constant force on the wire. Thus, the wire is free to move perpendicular to its direction, depending on lapping pressure or interaction with lapping particles. A damping force is applied, which is proportional to the velocity.

The material removal during the sawing process is implemented with a stepwise simulation. After simulating a process time period of $\Delta T$ the DEM simulation is stopped.

The ingot is partitioned in the circumferential direction of the wire, see Fig. 1. In these partitions the maximal normal contact forces have to be determined. For every discrete element in a partition the maximal normal contact forces have to be found. The maximum normal force for an individual contact is defined as the maximum normal force at a point, where the critical force for material removal $F^{\text {crit }}=3 \mathrm{mN}$ is exceeded until the force falls below $1 \mathrm{mN}$. This minimum value of critical force required to break material out was given in Möller [11]. According to the same author [11] the volume removed by a single contact with the normal force $F$ is

$V_{0}(F)=\frac{\pi \alpha^{\frac{1}{2}} \beta^{\star 2}}{2 \tan \phi H^{\frac{1}{2}}} F^{\frac{4 n+1}{2}}$

with the hardness $H$ and the tip half-angle of the indenter $\phi . \alpha, \beta^{\star}$ and $n$ are constants, which depend on the shape of the indenter and other factors. As in this work the aim is to calculate an average material removal rate and the material 
removal of an individual contact is of minor importance, the same average values for $\phi, \alpha, \beta^{\star}$ and $n$ can be used for all contacts. From all maximal normal contact forces $F^{i}$ in a partition the material removal rate $r$ can be calculated by averaging over the surface $A$ of the partition and the simulation time period $\Delta T$.

$r=\frac{1}{A \Delta T} \sum_{i} V_{0}\left(F^{i}\right)$

In a next step a scaling factor $s$ is calculated from the maximal material removal rate $r^{\max }$ of all partitions in the current simulation step, the simulation time $\Delta T$ and a specified maximal material removal depth $\Delta u^{\star}$ for one simulation step.

$s=\frac{\Delta u^{\star}}{r^{\max } \Delta T}$

The material removal depth for one partition is defined as

$\Delta u=r s \Delta T$.

Then the ingot geometry is updated by moving the discrete elements radially outwards with the distance $\Delta u$ of the partition. Elements in the circular part of the ingot have to be widened at the same time, so that the ingot maintains a continuous body. The use of the scaling factor insures, that in all simulation steps the maximal material removal is $\Delta u^{\star}$. Due to the short process time of one simulation step the statistical spread of the calculated material removal rates is quite large. The scaling factor insures, that simulation steps with very large material removal in one partition do not lead to unrealistic large grooves in the ingot. On the other hand simulation steps with very low material removal rates in all partitions still have a visible contribution to the change of the ingot geometry.

The simulations are run until a point is reached, where the kerf width did not change significantly for 30 simulation steps. This means, in the last 30 steps a stationary, stabilized process is reached, which resembles the actual sawing process. Therefore, only these last 30 steps of the simulation are then evaluated in a post-processing, which is described in the following. The average material removal rates of the partitions in the last 30 simulation steps are calculated. To these local material removal rates a sinus function is approximated with the method of least squares in the circular part of the ingot (partition 9 to 33). If the material removal along the kerf contour follows a sinus function the shape of the kerf stays constant, which corresponds to a stationary sawing condition. The maximum of the sinus function is considered as the material removal rate of the sawing process. As the sawing is in a stationary state this material removal rate or sawing rate is equivalent to the feed rate.

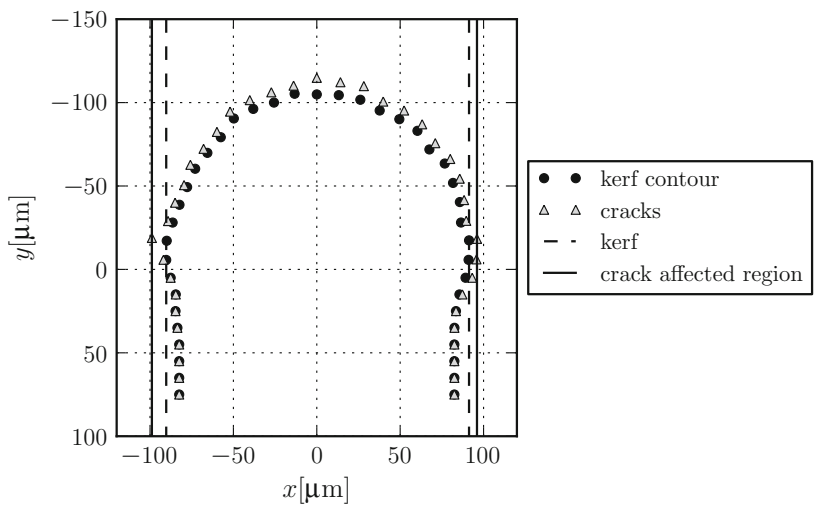

Fig. 2 Current ingot geometry and cracks at the end of the simulation for a wire speed of $5 \mathrm{~m} / \mathrm{s}$ and a lapping pressure of $0.1 \mathrm{MPa}$. The maximal extensions in $\mathrm{x}$-direction define the kerf width and the width of the crack affected region for the whole process

Usually in wire sawing the feed rate is an input parameter while the lapping pressure is a result of the feed rate and other process parameters, but in the simulation the lapping pressure is an input parameter and the feed rate or material removal rate is a result of the lapping pressure and other process parameters. This opposing approach does not affect the results.

From the maximal normal contact force in a partition the crack depth can be evaluated. Following Möller [11], the depth $l^{\mathrm{c}}$ of median cracks resulting from a force $F$ is

$l^{\mathrm{c}}=\left(\frac{\beta F}{K_{\mathrm{Ic}}}\right)^{\frac{2}{3}}$,

using the parameter $\beta=1 / 7$ and the fracture toughness $K_{\text {Ic }}=0.75 \mathrm{MPa} \sqrt{\mathrm{m}}$ for large grained polycrystalline silicon. As at the end of the simulation a stationary process stage is reached, the results can be used to estimate the kerf width and the width of the crack affected region for the whole process. As illustrated in Fig. 2, the kerf width is obtained from the largest extensions of the ingot geometry in $\mathrm{x}$-direction, and in the same way the crack affected region is obtained from the largest extensions of cracks in $\mathrm{x}$-direction.

The simulation region has a length of $1 \mathrm{~mm}$ in wire direction with periodic boundaries. And the wire diameter is $140 \mu \mathrm{m}$. The process time period in one simulation step is $\Delta T=25 \mu \mathrm{s}$ and the maximal material removal depth for one simulation step is $\Delta u^{\star}=2 \mu \mathrm{m}$. If nothing else is mentioned in the text the velocity of the wire is $5 \mathrm{~m} / \mathrm{s}$ and the abrasive particles have a size distribution of F800 according to norm DIN ISO 8486-2. The used material parameters for ingot ( $\mathrm{Si})$, abrasive particles $(\mathrm{SiC})$ and wire (steel) can be found in Table 1. The parameters for the indentation fracture model are given in Table 2. 
Table 1 Material parameters

\begin{tabular}{llll}
\hline Material & $\begin{array}{l}\text { Young's modulus } \\
E(\mathrm{GPa})\end{array}$ & Poisson's ratio $v$ & Density $\rho\left(\frac{g}{\mathrm{~cm}^{3}}\right)$ \\
\hline $\mathrm{Si}$ & 165 & 0.4 & 2.33 \\
$\mathrm{SiC}$ & 450 & 0.3 & 3.21 \\
Steel & 210 & 0.3 & 7.85 \\
\hline
\end{tabular}

Table 2 Parameters for the calculation of material removal $\left(\alpha, \beta^{\star}, H\right.$ and $n$ see [11])

\begin{tabular}{lllll}
\hline$\alpha$ & $\beta^{\star} \mu \mathrm{m} / \mathrm{N}^{n}$ & Half-angle $\phi$ & Hardness $H(\mathrm{GPa})$ & $n$ \\
\hline 2.0 & 13.76 & $70^{\circ}$ & 10.9 & 0.85
\end{tabular}

\section{Influence of particle concentration}

Although the particle concentration in the slurry before entering the sawing channel is well known, it is hard to obtain information about the particle concentration in the sawing channel. There are several possibilities which could change the particle concentration. At the point where the wire enters the ingot particles might get wiped off. Also air could enter the sawing channel and thereby change the particle concentration. In the sawing channel particles might leave the process zone. Unfortunately as far as the authors know there are no measurements of the particle concentration during sawing so far. In general in these simulations 4800 abrasive particles are placed in the process zone. The particle volume fraction in the initial state is $7.67 \%$. This seems rather low, but during the simulation the wire moves towards the ingot. Thus, the volume between wire and ingot decreases and the volume fraction of the abrasive particles increases. In order to investigate the influence of this parameter additional simulations where performed with 7200 and 9600 abrasive particles. The volume fractions of the particles in the initial state was 11.56 and $15.14 \%$. Since the volume fraction during the simulation is higher than in the initial state and the real volume fraction is expected to be lower than in the initial slurry these values are expected to be in a realistic range. The results are presented in Figs. 3 and 4. The results of all three simulations are in the same range. No dependency of the results on the particle number can be concluded. This can be explained by the observation, that in general the largest particles induce material removal. Thus, when increasing the overall particle number, the number of particles actually removing material stays constant. This leads to the conclusion, that this unknown process parameter has minor influence on the results and thus an exact knowledge of the particle concentration is not essential. Even if there might be some error in the choice of the

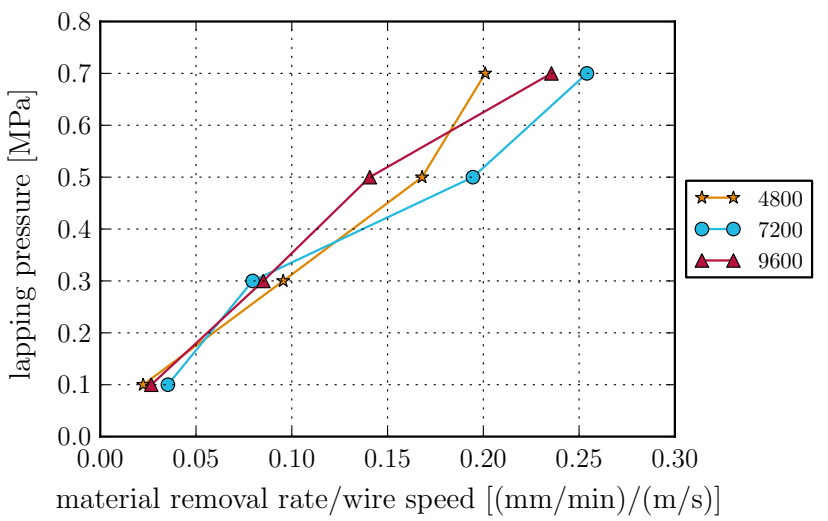

Fig. 3 Lapping pressure versus material removal rate/wire speed for different particle concentrations

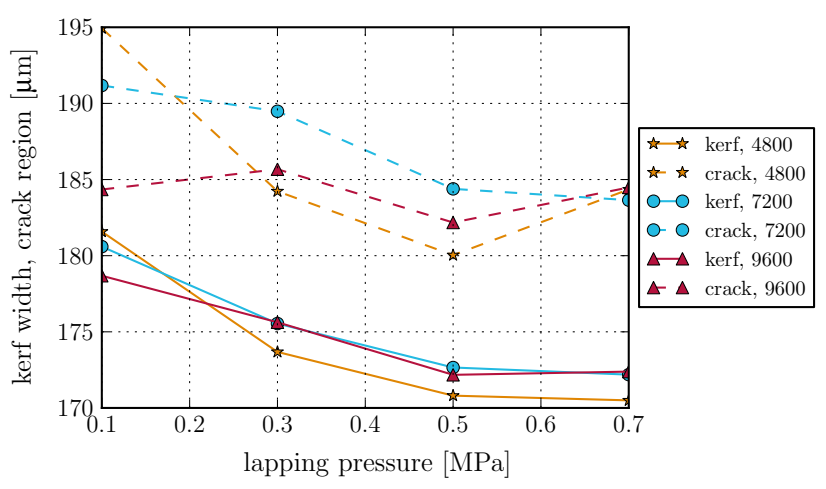

Fig. 4 Kerf width and width of crack affected region versus lapping pressure for different particle concentrations

particle concentration, the presented simulations are valuable.

\section{Influence of wire speed and lapping pressure}

In order to investigate the influence of wire speed and lapping pressure on the wire sawing process, simulations were performed with a wire speed of 5, 10, 15 and $20 \mathrm{~m} / \mathrm{s}$ and a lapping pressure of $0.1,0.3,0.5$ and $0.7 \mathrm{MPa}$. Figure 5 shows the geometrical configuration at the start and at the end of the simulation for a wire speed of $5 \mathrm{~m} / \mathrm{s}$ and a lapping pressure of $0.1 \mathrm{MPa}$. Figure 6 shows the kerf width with respect to the number of simulation steps for a wire speed of $5 \mathrm{~m} / \mathrm{s}$. It can be seen, that at the beginning of the simulation the kerf width increases. After several simulation steps a stationary state is reached, where the kerf width is almost constant. This final kerf width is considered as the realistic kerf width for the given conditions. As mentioned before, only this stationary state is evaluated in post-processing. In this figure it can also be seen, that the kerf width decreases with increasing lapping pressure. Figure 7 shows the material removal rate 

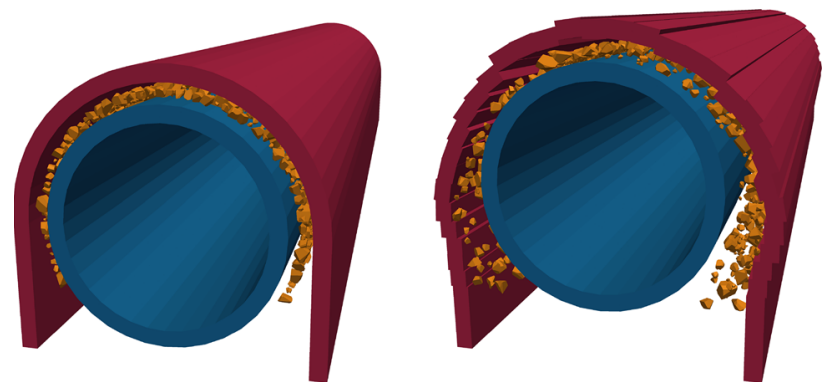

Fig. 5 Initial (left) und final (right) configuration for a wire speed of $5 \mathrm{~m} / \mathrm{s}$ and a lapping pressure of $0.1 \mathrm{MPa}$

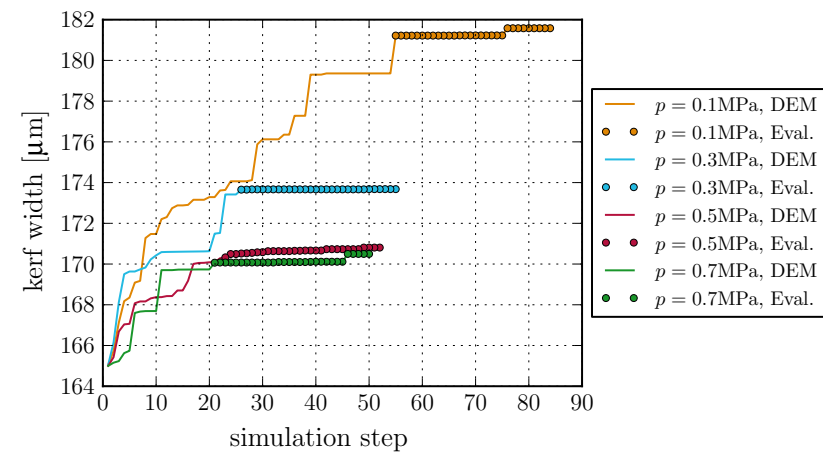

Fig. 6 Kerf width with respect to the number of simulation steps for a wire speed of $5 \mathrm{~m} / \mathrm{s}$. The region used for post-processing is marked with dots

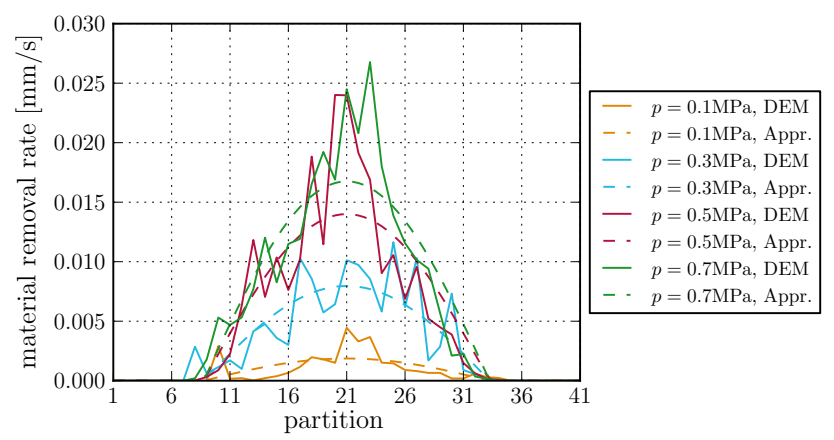

Fig. 7 Material removal rate of the partitions for a wire speed of $5 \mathrm{~m} / \mathrm{s}$. Solid lines show the results of the DEM simulation. Dashed lines show an approximated sinus function

of the partitions for a wire speed of $5 \mathrm{~m} / \mathrm{s}$ and the approximated sinus function. Although the material removal rates show some numerical spread it can be seen, that the average behaviour follows a sinus function.

Figure 8 shows the lapping pressure with respect to material removal rate/wire speed. The DEM results are compared to experimental results by Liedke [3]. Qualitatively the same behaviour can be seen in the simulation and in the experiment. The material removal rate increases with wire speed and lapping pressure. This is in accordance with the law of Preston [13]. The quantitative divergence between experiment and simulation might be due to some unknown process

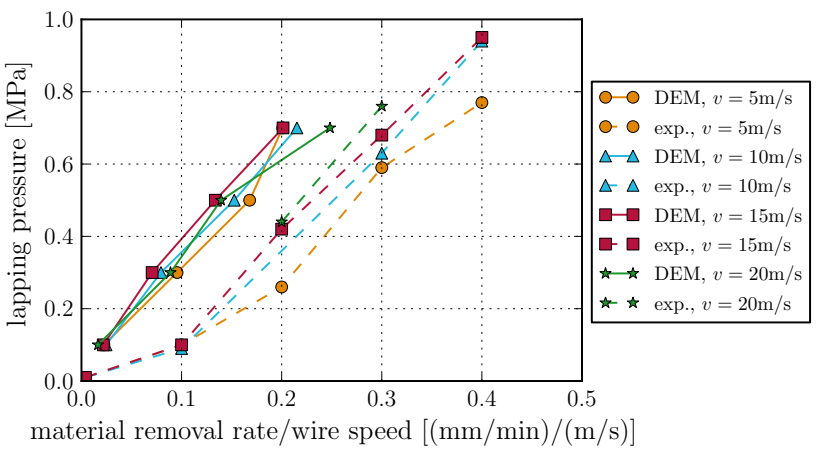

Fig. 8 Lapping pressure versus material removal rate/wire speed for different wire speeds

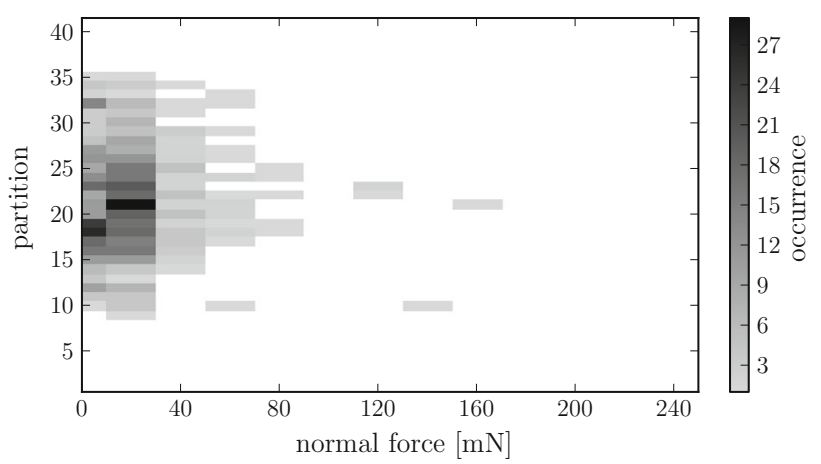

Fig. 9 Normal contact forces for a wire speed of $5 \mathrm{~m} / \mathrm{s}$ and a lapping pressure of $0.1 \mathrm{MPa}$

parameters of the experiment like particle shape or particle concentration in the sawing channel. The simulations also provide the possibility to take a detailed look at the individual interaction forces between abrasive particles and ingot. Figures 9 and 10 show the normal contact forces for a wire speed of $5 \mathrm{~m} / \mathrm{s}$ and a lapping pressure of 0.1 and $0.7 \mathrm{MPa}$. An increase in lapping pressure leads to more frequent contacts as well as higher contact forces. Figure 11 shows the kerf width and the width of the crack affected region with respect to the lapping pressure for different wire speeds. Except a slight decrease in kerf width with increasing lapping pressure no clear dependencies can be concluded.

\section{Influence of particle size}

An important parameter in wire sawing is the size of the abrasive particles. Simulations where performed with different size distributions ranging from larger to smaller size F400, F500, F600 and F800 according to norm DIN ISO 8486-2. In order to make the results comparable, the particle concentration has to be constant. Here the area fraction of particles is better used than volume fraction, as the distance between wire and ingot in the stationary state depends on the parti- 


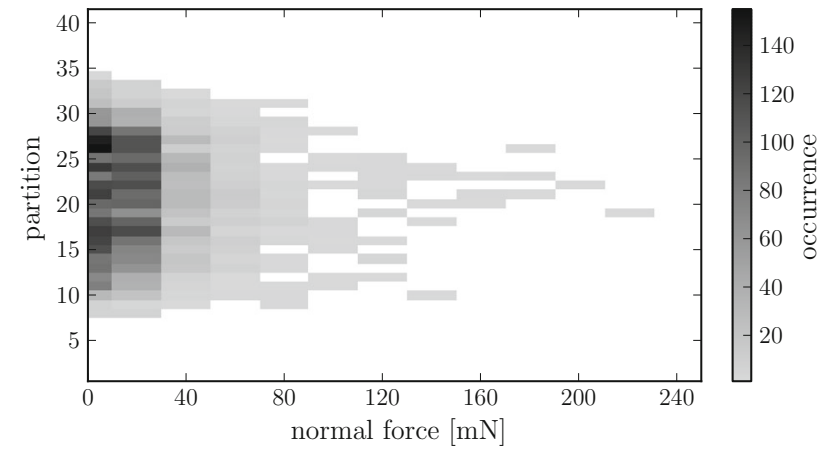

Fig. 10 Normal contact forces for a wire speed of $5 \mathrm{~m} / \mathrm{s}$ and a lapping pressure of $0.7 \mathrm{MPa}$ for particles with a size distribution of F800

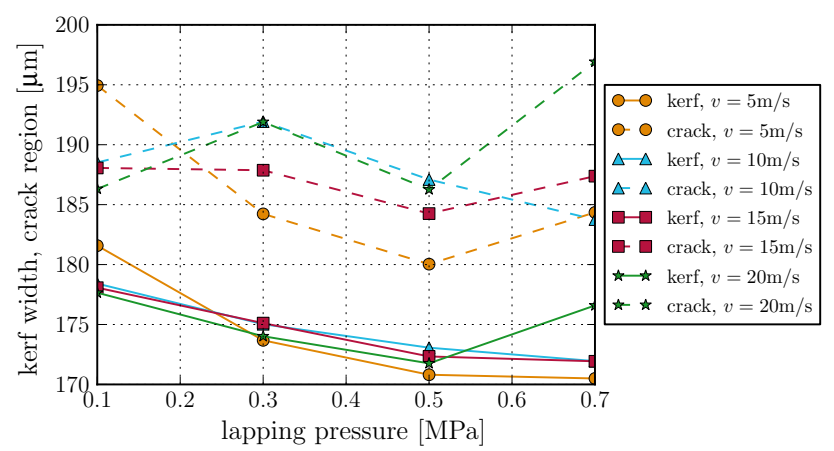

Fig. 11 Kerf width and width of crack affected region versus lapping pressure for different wire speeds

cle size and is unknown in advance. This is realized by the relation

$\frac{\pi d_{\mathrm{s} 50}^{2} n^{\mathrm{P}}}{4 A^{\mathrm{w}}}=$ const.

where $d_{\mathrm{s} 50}, n^{\mathrm{P}}$ and $A^{\mathrm{w}}$ are the mean particle size, the number of particles and the surface area of the wire, respectively. Thus, for larger abrasive particles the particle number is reduced. The results are presented in Figs. 12 and 13. It is clearly visible, that larger particles lead to higher material removal rates. This is in accordance with experimental results by Skomedal et al. [14] and Retsch et al. [15]. As expected, the kerf width increases with the particle size. Furthermore, the difference between the width of the kerf and the crack affected region increases with the particle size. This means that deeper cracks will remain in the wafer after sawing, when larger particles are used. In Fig. 14 the normal contact forces for a wire speed of $5 \mathrm{~m} / \mathrm{s}$ and a lapping pressure of 0.7 MPa for particles with a size distribution of F400 are shown. Compared with the results for a finer particle distribution of F800 in Fig. 10 it can be seen, that simulations with larger particles lead to less frequent contacts but higher forces, which cause deeper cracks. This is in accordance with experimental results by Bidiville et al. [16].

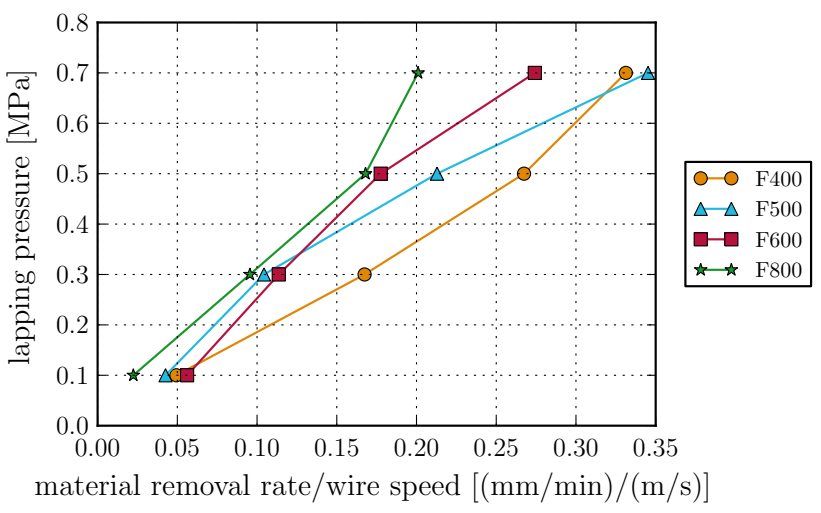

Fig. 12 Lapping pressure versus material removal rate/wire speed for different particle sizes

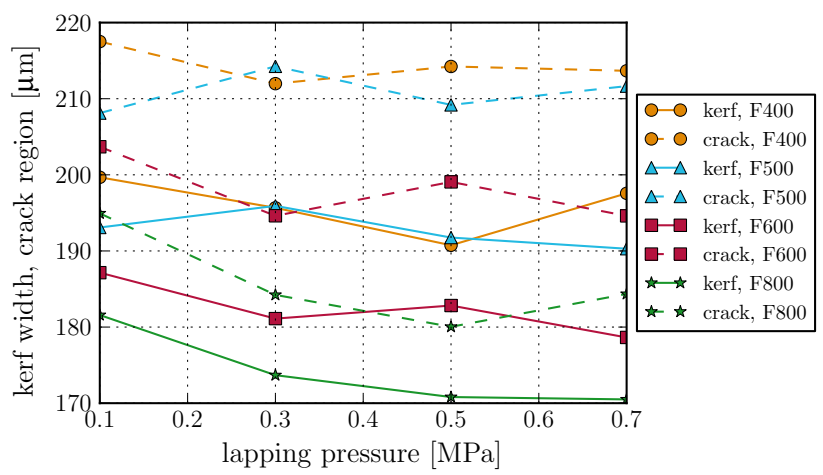

Fig. 13 Kerf width and width of crack affected region versus lapping pressure for different particle sizes

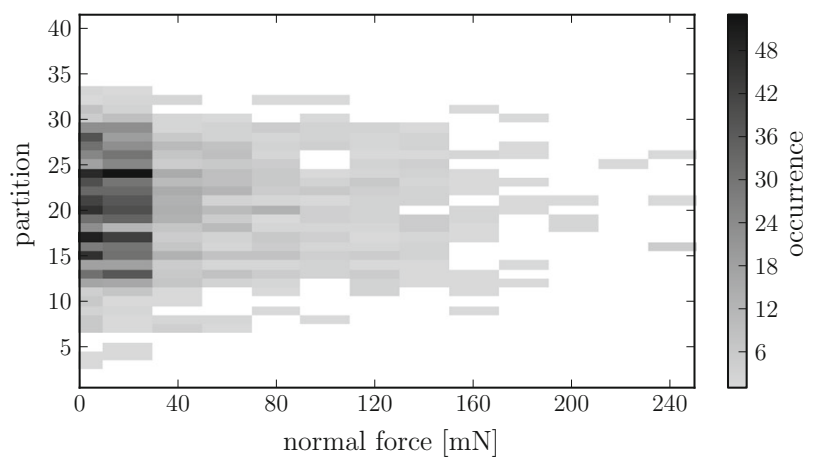

Fig. 14 Normal contact forces for a wire speed of $5 \mathrm{~m} / \mathrm{s}$ and a lapping pressure of $0.7 \mathrm{MPa}$ for particles with a size distribution of F400

\section{Influence of particle shape}

In general the particles in the simulation have a random shape which is produced by starting with a cube and adding random cutting planes [9]. If starting with a prolate $(2 \times 1 \times 1)$ or oblate $(2 \times 2 \times 1)$ cuboid and with little cutting depth of the random cutting planes, particles are obtained, which are random, but tend to have a prolate or oblate shape. Simulations where performed with these three shapes (normal, prolate 


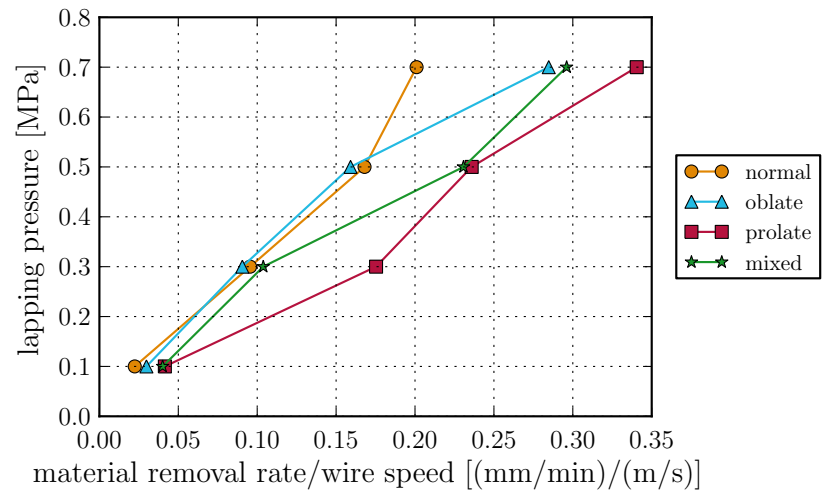

Fig. 15 Lapping pressure versus material removal rate/wire speed for different particle shapes

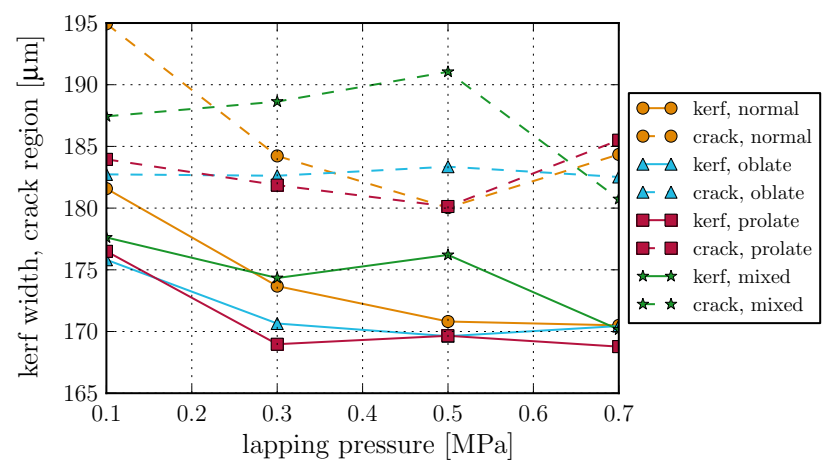

Fig. 16 Kerf width and width of crack affected region versus lapping pressure for different particle shapes

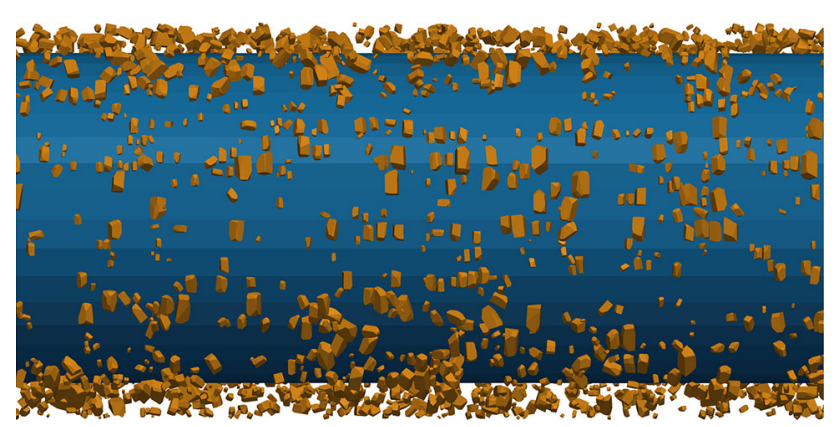

Fig. 17 Alignment of prolate particles perpendicular to the wire axis

and oblate) and a mixture of the three particle shapes. The results are shown in Figs. 15 and 16. It can clearly be seen, that the particle shape has a strong influence on the sawing process. The favourable particle shape is the prolate shape. In this case the highest material removal rates are obtained, while the width of the kerf and the crack affected region is minimal. Figure 17 shows the wire and the prolate particles at the end of the simulation. It can be seen, that during the simulation the particles align themselves perpendicular to the wire axis, so that an optimal rolling motion is obtained.
This rolling motion leads to more frequent contacts between abrasive particles and ingot, which explains the high material removal rates. Furthermore, the alignment reduces the minimal distance between wire and ingot, thus producing the small kerf width.

\section{Influence of debris}

During wire sawing the amount of silicon debris in the slurry is increasing since silicon is removed. This changes the properties of the slurry during the sawing process. Bidiville et al. [17] presented experiments investigating the influence of the amount of debris in the slurry on the sawing process. They noticed, that an increasing amount of debris leads to increasing wafer roughness, lower fracture strength of the wafers and the apparition of saw marks. In order to better understand the reason for this observations simulations were performed without silicon debris and with additional silicon debris of a volume equalling 5.67 and $11.37 \%$ of the volume of $\mathrm{SiC}$. A wire length of $500 \mu \mathrm{m}$ is simulated. The results are shown in Figs. 18 and 19. It can be seen, that a higher amount of debris leads to higher material removal rates. Also the width of the crack affected region increases. In Figs. 20 and 21 the normal contact forces for a wire speed of $5 \mathrm{~m} / \mathrm{s}$ and a lapping pressure of $0.5 \mathrm{MPa}$ for a slurry with no debris and a slurry with a debris volume equalling $11.37 \%$ of the volume of $\mathrm{SiC}$ are shown. The results show, that the forces are higher when a slurry containing debris is used. This explains the higher material removal rates. This result also agrees with the higher roughness and lower fracture toughness of the wafer observed by Bidiville et al. [17] when using slurry with a high amount of debris. The higher material removal rates for slurries containing debris are similar to the experimental results of Bhagavat et al. [18]. They added smaller abrasive particles to the slurry and obtained higher material removal rates, compared to slurry only containing large abrasive particles.

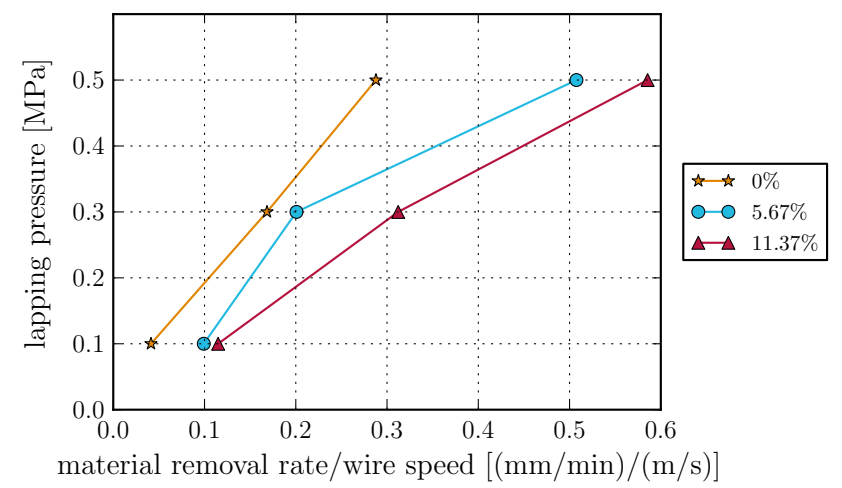

Fig. 18 Lapping pressure versus material removal rate/wire speed for different amounts of debris 


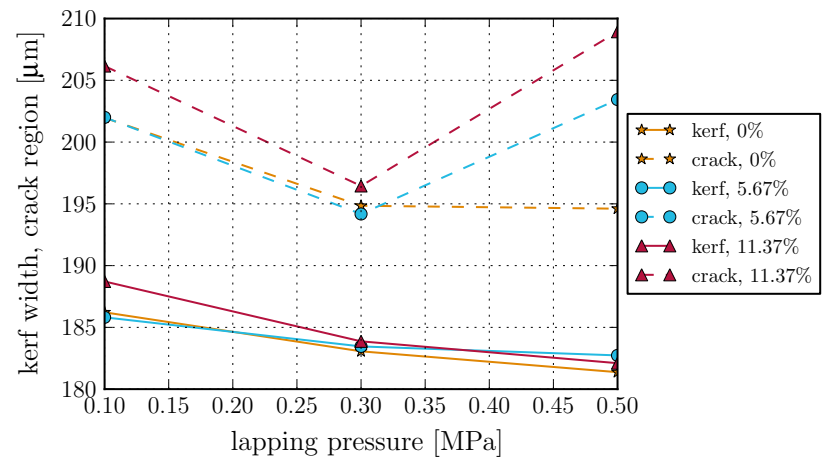

Fig. 19 Kerf width and width of crack affected region versus lapping pressure for different amounts of debris

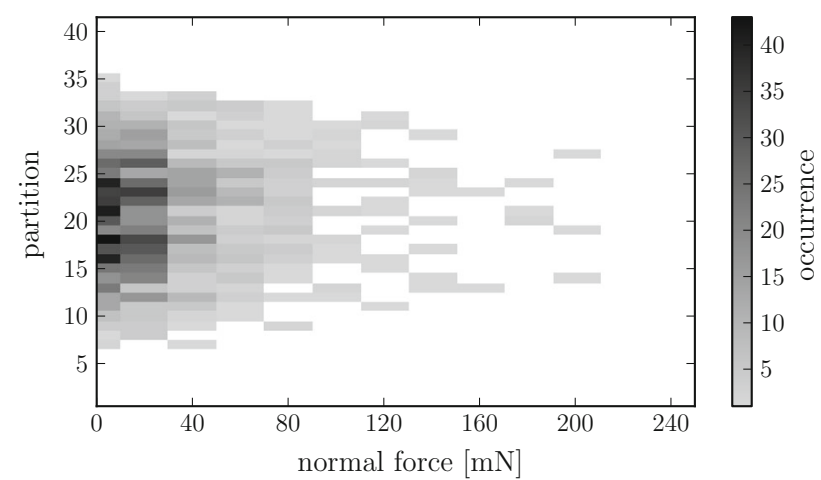

Fig. 20 Normal contact forces for a wire speed of $5 \mathrm{~m} / \mathrm{s}$ and a lapping pressure of $0.5 \mathrm{MPa}$ for a slurry with no debris

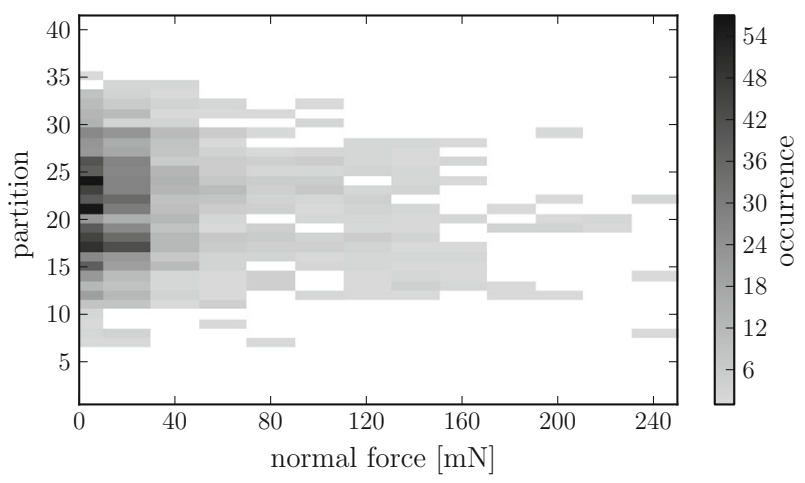

Fig. 21 Normal contact forces for a wire speed of $5 \mathrm{~m} / \mathrm{s}$ and a lapping pressure of $0.5 \mathrm{MPa}$ for a slurry with a debris volume equalling $11.37 \%$ of the volume of $\mathrm{SiC}$

\section{Conclusion}

In the present paper, a new approach in modeling the wire sawing process at micro level was presented. The abrasive particles were simulated by convex polyhedra. These polyhedra provide a good representation of the shape of real abrasive particles. By means of extensive DEM simulations, the particle motion and the interaction forces between particles and ingot are computed. The local material removal during the sawing process was taken into account by a model based on indentation cracking. The calculated local material removal rates were used to update the shape of the ingot in a stepwise simulation. From the results of the simulation at micro level, the macroscopic process parameters kerf width, material removal rate and the width of the crack affected region were evaluated in a post-processing step. The model was applied to study the influence of different aspects on the sawing process. First of all, the material removal rate increases with increasing lapping pressure and increasing wire speed. Larger particles lead to higher material removal rates but at the cost of larger kerf width and larger crack affected region due to higher forces. The particle shape has a significant influence on the sawing process. Prolate particles are found to have the best characteristics as they lead to a high material removal rate, small kerf width and small crack affected region at the same time. When debris is present in the slurry higher forces occur, which explains the higher roughness and lower fracture toughness in the experiments of Bidiville et al. [17].

Acknowledgments This work was funded by the German Research Foundation in the project KU 929/19.

\section{References}

1. Ishikawa KI, Suwabe H, Itoh SI (2003) Study on slurry actions in slicing groove and slicing characteristics at multi-wire saw. In: ASPE proceedings

2. Kaminski S, Wagner T, Rietzschel R, Fütterer W, Funke C, Möller HJ (2010) The role of the slurry transport in multi-wire sawing of solar silicon wafers. In: 25rd European photovoltaic solar energy conference and exhibition, pp 1312-1317

3. Liedke T (2011) Makro- und mikromechanische Untersuchungen zum Drahtsägen von Siliziumwafern. Ph.D. thesis, TU Bergakademie Freiberg, Institut für Mechanik und Fluiddynamik

4. Liedke T, Kuna M (2013) Discrete element simulation of micromechanical removal processes during wire sawing. Wear 304(12):77-82

5. Wagner T, Möller HJ (2008) A 3d wire saw model of the slurry flow to predict forces exerted upon silicon ingots during cutting. In: $23 \mathrm{rd}$ European photovoltaic solar energy conference and exhibition, pp $1315-1320$

6. Bierwisch C, Kübler R, Kleer G, Moseler M (2011) Modelling of contact regimes in wire sawing with dissipative particle dynamics. Philos Trans R Soc A 369(1945):2422-2430

7. Nassauer B, Hess A, Kuna M (2014) Numerical and experimental investigations of micromechanical processes during wire sawing. Int J Solids Struct 51(14):2656-2665

8. Nassauer B, Kuna M (2013) Contact forces of polyhedral particles in discrete element method. Granul Matter 15(3):349-355

9. Nassauer B, Liedke T, Kuna M (2013) Polyhedral particles for the discrete element method: geometry representation, contact detection and particle generation. Granul Matter 15(1):85-93

10. Nassauer B (2015) DEM-Simulation mikromechanischer Abtragsprozesse bei der Werkstoffbearbeitung mit fluidgekoppelten Partikelsystemen. Ph.D. thesis, TU Bergakademie Freiberg, Institut für Mechanik und Fluiddynamik 
11. Möller HJ (2004) Basic mechanisms and models of multi-wire sawing. Adv Eng Mater 6(7):501-513

12. Bestimmung und Bezeichung der Korngröenverteilung - Teil 2: Mikrokörnungen F230 bis F1200 (1996)

13. Preston FW (1927) The theory and design of plate glass polishing machines. J Soc Glass Technol 11:214-256

14. Skomedal G, Øvrelid EJ, Armada S, Espallargas N (2011) Effect of slurry parameters on material removal rate in multi-wire sawing of silicon wafers: a tribological approach. Proc Inst Mech Eng Part J 225(10): 1023-1035

15. Retsch S, Jentsch S, Möller HJ (2012) Influence of shape and size distribution of abrasives on the cutting performance of multi wire sawing and lapping. In: 27rd European photovoltaic solar energy conference and exhibition, pp 947-950
16. Bidiville A, Wasmer K, Michler J, Nasch PM, Van Der Meer M, Ballif C (2010) Mechanisms of wafer sawing and impact on wafer properties. Prog Photovolt 18(8):563-572

17. Bidiville A, Neulist I, Wasmer K, Ballif C (2011) Effect of debris on the silicon wafering for solar cells. Sol Energy Mater Sol Cells 95(8):2490-2496

18. Bhagavat S, Liberato JC, Chung C, Kao I (2010) Effects of mixed abrasive grits in slurries on free abrasive machining (fam) processes. Int J Mach Tools Manuf 50(9):843-847 